ARTICLE

\title{
Natural selection increases female fitness by reversing the exaggeration of a male sexually selected trait
}

\author{
Kensuke Okada (1) 1,6, Masako Katsuki ${ }^{2,6}$, Manmohan D. Sharma³, Katsuya Kiyose ${ }^{1}$, Tomokazu Seko ${ }^{4}$, \\ Yasukazu Okada (10 ${ }^{5}$, Alastair J. Wilson ${ }^{3} \&$ David J. Hosken (10 ${ }^{3 凶}$
}

Theory shows how sexual selection can exaggerate male traits beyond naturally selected optima and also how natural selection can ultimately halt trait elaboration. Empirical evidence supports this theory, but to our knowledge, there have been no experimental evolution studies directly testing this logic, and little examination of possible associated effects on female fitness. Here we use experimental evolution of replicate populations of broad-horned flour beetles to test for effects of sex-specific predation on an exaggerated sexually selected male trait (the mandibles), while also testing for effects on female lifetime reproductive success. We find that populations subjected to male-specific predation evolve smaller sexually selected mandibles and this indirectly increases female fitness, seemingly through intersexual genetic correlations we document. Predation solely on females has no effects. Our findings support fundamental theory, but also reveal unforseen outcomes-the indirect effect on females-when natural selection targets sex-limited sexually selected characters.

\footnotetext{
${ }^{1}$ Graduate School of Environmental and Life Science, Okayama City, Okayama, Japan. ${ }^{2}$ Department of Agricultural and Environmental Biology, The University of Tokyo, Bunkyo-ku, Tokyo, Japan. ${ }^{3}$ Centre for Ecology \& Conservation, University of Exeter, Penryn, UK. ${ }^{4}$ National Agriculture and Food Research Organization, Central Region Agricultural Research Center, Tsukuba, Ibaraki, Japan. ${ }^{5}$ Department of Biological Sciences, School of Science, Tokyo Metropolitan University, Hachiohji, Tokyo, Japan. ${ }^{6}$ These authors contributed equally: Kensuke Okada, Masako Katsuki. ${ }^{凶}$ email: d.j.hosken@exeter.ac.uk
} 
S exual selection typically acts more strongly on males and is responsible for the evolution of a vast array of exaggerated characters that enhance male sexual fitness components ${ }^{1-4}$. Lande's ${ }^{5}$ and Kirkpatrick's ${ }^{6}$ models of sexual selection via the Fisher $^{7}$ process-the null models of intersexual selection ${ }^{8}$ clearly shows how this can occur. They also demonstrate how natural selection can oppose sexual selection as trait values move beyond their naturally selected optima (reviewed in ref. ${ }^{9}$ ). While theory is clear on the joint effects of natural and sexual selection on sexual trait evolution, explicit experimental tests of theoretical predictions are required to fully understand sexual trait evolution ${ }^{10}$.

One potentially important source of natural selection that could affect the evolution of sexually selected traits is predation ${ }^{1}$, and many studies have shown predation can seemingly oppose the exaggeration of male sexual characters. For example, sexual signals are conspicuous to potential mates but may also attract predators and parasitoid ${ }^{11}$. This is particularly well documented in orthopterans and frogs ${ }^{12-18}$, and this form of natural selection is probably responsible for the loss of cricket sexual signals on two Hawaiian islands ${ }^{19,20}$. More generally, predation appears to reverse the evolution of extreme sexually selected phenotypes (reviewed in the ref. ${ }^{21}$ ) and males frequently reduce their sexual signaling in response to predation risk, which can result in decreased mating success when risk is high ${ }^{22-25}$. Nonetheless, while there is ample evidence that predation selects against sexual trait enhancement, there is limited direct experimental verification that this selection causes evolutionary responses in these traits (but see e.g. ${ }^{26,27}$ ).

Female reproductive success can also be impacted by predation $^{28,29}$. For example, egg-carrying females can be slower ${ }^{30,31}$ and suffer higher predation rates ${ }^{29,32}$. Resultant anti-predator behaviors may reduce foraging efficiency and reproductive activity, and thus be costly to females (reviewed in the ref. ${ }^{22}$ ). Costs can accrue via delayed development, slower growth or postponed reproduction $^{33-36}$. Nonetheless, while there is ample evidence that predation selects on both females and males, the joint action of selection on the sexes is frequently investigated independently. Unfortunately, without exploring how predation affects both sexes, we are unlikely to fully understand how predation affects sexual trait evolution ${ }^{10}$. This is especially true when intersexual genetic correlations link sexually selected male characters with female fitness, because selection on one sex can affect the other through these correlations $s^{37,38}$. And again, controlled experimental tests for evolutionary responses to predation are usually not undertaken.

Here, we use the broad-horned flour beetle Gnatocerus cornutus to directly test for effects of predation on the evolution of a male sexually selected character (their mandibles) and female lifetime reproductive success (LRS). The enormously enlarged male mandibles are used in male-male fights, and males with larger mandibles have higher fighting and mating success ${ }^{39-42}$. Females lack this exaggerated character completely ${ }^{40,43}$. Previous work has shown that males with larger mandibles sire daughters with lower fecundity, and that directly selecting for increased (or decreased) mandible size results in decreased (or increased) female fitness (LRS) ${ }^{40,42}$. This apparently occurs because of the beetle's genetic architecture, which means evolving larger mandibles results in the correlated evolution of masculinized females (even though females never develop mandibles). Basically, the enlarged male mandible requires a masculinized head and prothorax to function optimally and this means males with larger mandibles have smaller abdomens ${ }^{40,44}$. Thus although females never develop mandibles, selecting on male mandibles ultimately affects female abdomen size, which likely determines the number of eggs a female carries ${ }^{40,45}$.

These previous $G$. cornutus studies clearly document intralocus sexual conflict over beetle morphology 40,42 and point to a negative intersexual correlation between (male) mandible size and female fitness, although this link has not been directly established and requires confirmation (c.f. ${ }^{46}$ ). With this in mind we investigate the beetle's intersexual genetic architecture for key focal traits using a standard pedigree-based mixed model approach (commonly referred to as an animal model $)^{47}$. We also establish replicate (3/treatment) experimental evolution populations subjected to either male or female predation, along with control populations ( $n=3$, for nine total populations) to investigate how predation affects an exaggerate male trait and female fitness. The assassin bug Amphibolus venator, which preys on various storedproduct insect pests including flour beetles ${ }^{48}$, is the model predator. After eight generations of experimental evolution, we measure female fitness (LRS) and a range of morphological characters, including mandible size. Morphology was also measured during experimental evolution. We find strong effects of male-specific predation on morphology and female fitness, while predation on females alone had no effects. We additionally test for associations between male mandible size and predation likelihood and find larger mandibles result in greater likelihood of predation, and also show that evolving under predation made males less effective fighters.

\section{Results}

Genetic architecture. The genetic parameters estimated from the animal model analyses confirmed what previous experimental evolution studies had only inferred ${ }^{39,40}$. Likelihood ratio comparisons of univariate models confirmed the presence of additive genetic variance in all four traits (Supplementary Tables 1-2) as expected. Note that the decision to treat the two mass measures (body/abdomen) as the same trait across the sexes was justified by an absence of significant genotype-by-sex $(\mathrm{GxS})$ interactions in univariate models-a GxS implies sex limited genetic variance is present and can manifest as cross-sex genetic correlations $\left(r_{\mathrm{Gmf}}\right)$ $<+1$ and/or differences in sex specific additive genetic variances. Here $r_{\mathrm{Gmf}}$ was approximately +1 for both traits, but we do note there is a qualitative pattern of higher additive variance for body mass in females (see Supplementary Tables 1 and 2). Multivariate models further confirmed the presence of additive genetic variance (LRT comparison of null model to one with a diagonal genetic matrix; $\chi^{2}{ }_{4}=74.96, P<0.0001$ ), as well as significant among-trait genetic correlation structure (LRT comparison of a model with a diagonal genetic matrix with all genetic correlations are fixed to zero to the full model in which all genetic correlations are estimated; $\left.\chi_{6}^{2}=39.02, P<0.0001\right)$. Parameter estimates from the full multivariate model show substantial genetic variation in all traits measured and reasonably high trait heritability (Table 1). All traits were positively genetically correlated except male mandible size and female fitness (lifetime reproductive success) and male mandible size and abdominal mass, which were both strongly negatively correlation. Individual genetic correlations were nominally significant at $\alpha=0.05$ (based on $\left|r_{\mathrm{G}}\right|>1.96 \mathrm{SE}$ ) except those between body and abdomen mass and between body mass and female LRS. The lack of correlation between body and abdomen mass is consistent with previous findings ${ }^{40}$.

Predation and evolution. The experimental treatments we imposed on the replicated beetle populations generated treatment specific evolutionary responses in some traits but not others, and responses were not linear (Fig. 1). When we compared trait values at the end of the experimental evolution period, we found that male predation significantly reduced male mandible size (Fig. 2a. Overall treatment effect: $F_{2,6}=31.07 ; \quad P<0.001$ : post-hoc $t$-tests [with sequential Bonferroni adjustment] revealed that when males were exposed to predators they evolved the smallest mandibles (all $P<$ 0.01 ), while the control and female treatments did not differ in 
Table 1 Estimates of the genetic variance-covariance structure among traits in the breeding design.

\begin{tabular}{|c|c|c|c|c|}
\hline Traits & Body mass & Abdominal mass & Male mandible size & Female LRS \\
\hline Body mass & $\mathbf{0 . 3 4 6}(0.085)$ & $0.296(0.164)$ & $\mathbf{0 . 5 7 4}(0.162)$ & $0.422(0.242)$ \\
\hline Abdominal mass & & $\mathbf{0 . 5 1 3}(0.096)$ & $-\mathbf{0 . 4 1 5}(0.171)$ & $0.596(0.199)$ \\
\hline Male mandible size & & & $\mathbf{0 . 3 8 0}(0.111)$ & $-\mathbf{0 . 5 9 8}(0.250)$ \\
\hline
\end{tabular}

mandible size $(P=0.36))$. Similarly, male predation resulted in increased male abdomen size (Overall treatment effect: $F_{2,6}=31.04$; $P<0.001)$ : post-hoc $t$-test revealed that males exposed to predators evolved the largest abdomens (all $P<0.01$ ) while control and female predator-exposure treatments did not differ $(P=1.0)$ (Fig. 2b). Total male body size was unaffected by our treatments (Fig. $2 \mathrm{c}: F_{2,6}$ $=1.17 ; P=0.373$ ). Finally, evolving under predation makes males less effective fighters $\left(F_{2,6}=24.364 ; P=0.0013\right)$ (Supplementary Fig. 1). Post-hoc $t$-tests showed that this was because in the male predation treatment, fighting success was lower (all $P<0.01$ ), while the other treatments did not differ (control $=$ female predation: $P$ $=0.190)$.

Predation also affected female LRS (Fig. 2d: $F_{2,6}=21.29$; $P=0.002)$ (note females mated with "non-evolved" tester males). Post-hoc $t$-tests showed that this was because in the male predation treatment female fitness was higher (all $P<0.01$ ), while the other treatments did not differ (control $=$ female predation: $P=0.54$ ). Thus exposing males to predators resulted in the evolution of smaller male mandibles and higher female fitness. As with males, female abdomen size also increased under male predation (Fig. 2e. Overall treatment effect: $\left.F_{2,6}=10.75 ; P=0.010\right)$ : post-hoc $t$-test revealed the male predator exposure group evolved the largest female abdomens (all $P \leq 0.01$ : control $=$ female predation $P=$ $1.0)$ ). Finally, female body size was unaffected by our experimental regimes (Fig. 2f. $F_{2,6}=1.65 ; P=0.269$ ).

Mandible size and predation likelihood. In the direct assessment of associations between mandible size and predation probability, 45 of 70 male $G$. cornutus were preyed by female $A$. venator within thirty minutes. The likelihood of being attacked by the predator was positively associated with mandible size (Fig. 3; Intercept $( \pm \mathrm{se})=-7.18 \pm 2.34 ; \chi^{2}=12.45 ; P<0.001$. Coefficient $( \pm$ se $\left.)=19.52 \pm 5.89 ; \chi^{2}=15.09 ; P<0.001\right)$. Possible reasons for this are discussed below.

Main results. To summarize the main findings: male-specific predation results in the evolution of an altered, more feminized male phenotype that includes reductions in the size of a malelimited sexually selected trait. Additionally, because of the beetle's genetic architecture, the "new" demasculinized male phenotype is transmitted through to the female phenotype and results in higher fitness females (Fig. 4).

\section{Discussion}

Predation is frequently invoked as an evolutionary brake on the exaggeration of sexually selected traits and there are many studies consistent with this logic, usually documenting selection against larger characters or assessing macro-evolutionary patterns consistent with it ${ }^{26,27}$. Here, we employ experimental evolution and directly demonstrate that male-specific predation not only reversed the exaggeration of a sexually selected trait, but additionally, this reversal results in higher female fitness. This boost to female fitness occurs because predators select against larger mandibles, and this results in a less masculinized beetle phenotype (including a larger abdomen), and because of shared genetic architecture across the sexes, females also become more feminized and produce more offspring. We discuss these findings in turn.

The likelihood of predation increases with male mandible size and when populations were forced to evolve with predation on males, smaller mandibles evolve. Thus increased natural selection via predation reduces the size of a sexually selected trait, which is broadly consistent with theory ${ }^{5,6}$ (reviewed in the ref. ${ }^{1}$ ). Previous work with $G$. cornutus suggests why predation has greater impact on males with larger mandibles. Mandible size is negatively phenotypically and genetically associated with locomotor activity $^{49}$, and locomotor activity (running) is a predator escape mechanism in flour beetles ${ }^{50}$. Reduced running and lower escape rates for males with large mandibles would explain the microevolutionary pattern we detect. Interestingly, there is no intersexual correlation for locomotion in this beetle ${ }^{49}$, so there was no expectation that predation on females should impact male running speed and hence morphology. Be that as it may, we clearly show that predation risk increases with mandible size and that this natural selection reverses the evolutionary exaggeration of a sexually selected male trait.

Females from the male-predation populations evolve higher fitness (higher LRS when mating with stock tester males) even though females were not directly exposed to predators. Previously Harano et al. ${ }^{40}$ demonstrated that directly artificially selecting for larger (male) mandibles reduced female fitness. This occurred because selection for increased mandible size resulted in a more masculinized phenotype, and Harano et al. ${ }^{40}$ suggested this masculinization rippled through inferred intersexually-shared genetic architecture to increase the masculinization of the female phenotype, thereby reducing female fitness. The intersexual genetic associations we document here, especially the negative male mandible-female LRS correlation, flesh out this explanation and confirm previous inference ${ }^{40}$. Negative intersexual fitness associations are common ${ }^{51,52}$ because alleles conferring high fitness to one sex frequently lower fitness in the other ${ }^{53,54}$. In addition to any sexually antagonistic selection on body morphology, male beetles with larger mandibles are also more aggressive toward females ${ }^{55}$. Thus exposure to males with larger mandibles potentially reduces female LRS due to (misdirected) male attacks ${ }^{55}$. Therefore, there could be two avenues to increased female fitness when predators select against large male mandibles, a reduction in ontogenetic conflict load (intralocus sexual conflict $\operatorname{load}^{37}$ ), and reduced male-to-female aggression. In any case, predation on males causes an evolutionary reduction in mandible size and this results in higher female fitness. Thus male-biased predation indirectly selects for an increase in female quality, which is an interesting outcome.

The net population level effects of sexual selection and sexual conflict over optimal phenotypes are not clear ${ }^{51,56}$, with for example, evidence that sexual selection can both increase and decrease population extinction rates ${ }^{57-59}$. Additionally, intralocus sexual conflict (as documented in the flour beetle ${ }^{40}$ ) is thought to 

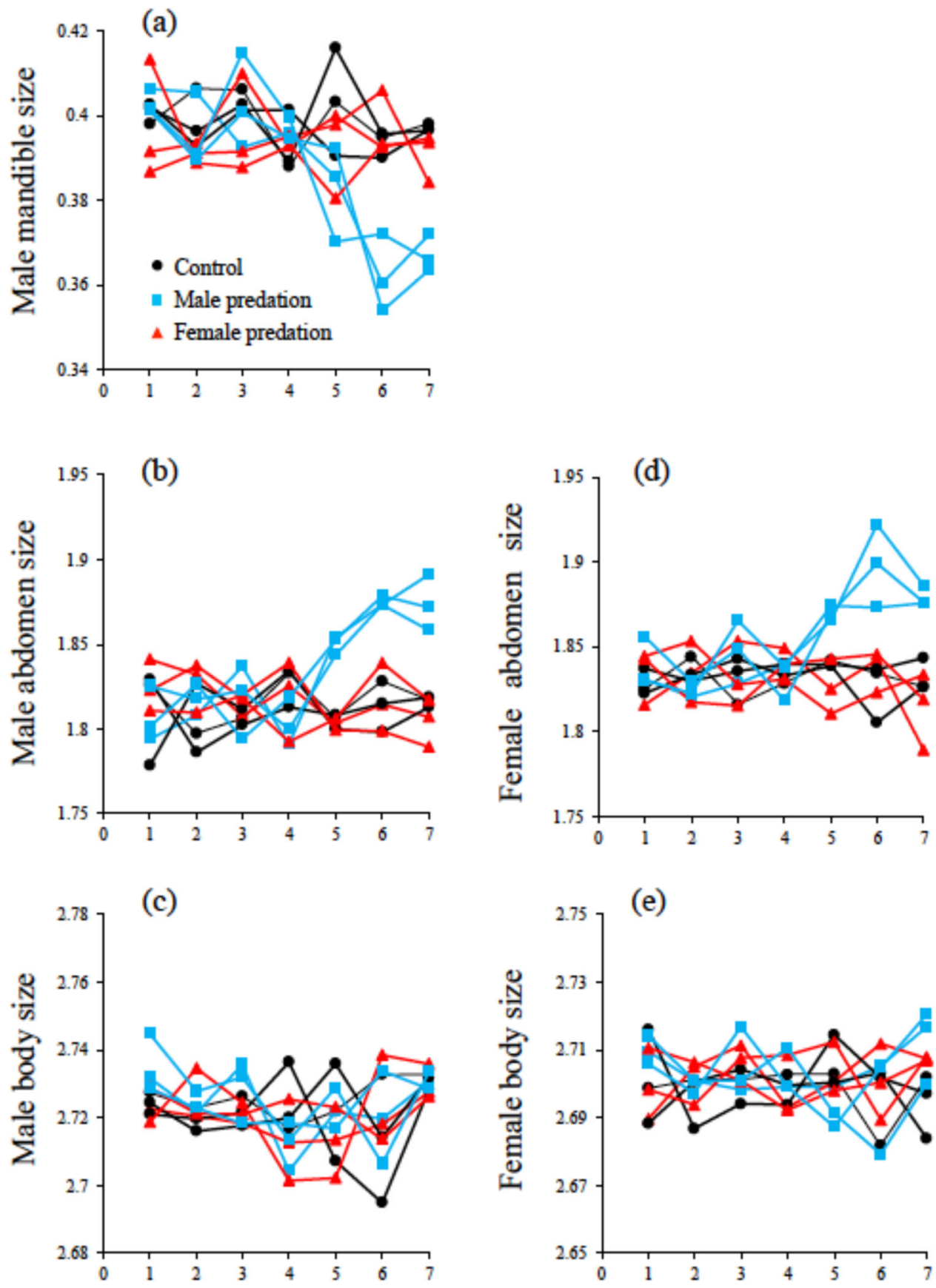

\section{Generation}

Fig. 1 Responses to sex-specific selection through predation over seven generations. Shown are male mandible size (mm) (a), male abdomen size $(\mathrm{mg})(\mathbf{b})$, male body size ( $\mathrm{mg})(\mathbf{c})$, female abdomen size $(\mathrm{mg})(\mathbf{d})$ and female body size ( $\mathrm{mg}$ ) (e) (population means). Black circles are the control populations that were not subjected to selection by predation. Blue squares and red triangles, are the populations with male and female exposure to predators, respectively. Note we did not measure female fitness (lifetime reproductive success: LRS) at every generation as it was not logistically possible. Population means (estimated by measuring 50 male and females per generation), for each population/treatment are shown because only populations evolve and thus population is the biologically relevant unit of replication in an experimental evolution study. Source data are provided as a Source Data file.

constrain population adaptation because sexually antagonistic selection keeps each sex from its fitness optima ${ }^{51,60}$. Our results suggest that predator removal of males with the largest sexual traits reduces intralocus (ontogenetic) sexual conflict costs, enhancing female reproductive performance, which should (all else being equal) increase population productivity. Relaxing other sexual conflict can also increase population fitness ${ }^{61,62}$. It is interesting to note that predators are usually seen as suppressing prey populations ${ }^{63}$, and this can have indirect benefits for ecological competitors of the prey taxon (predation on one species 
(a)

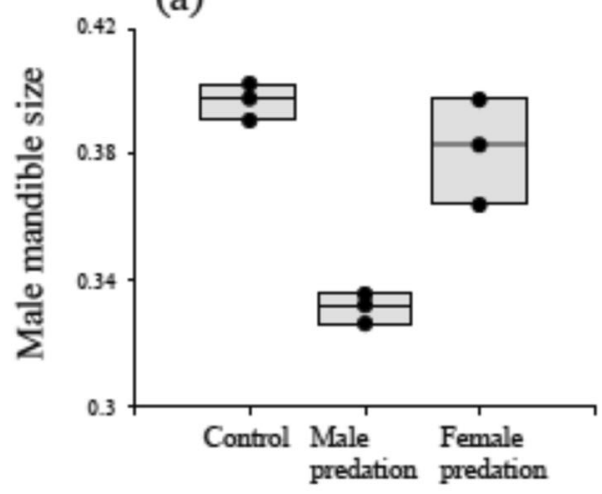

(b)

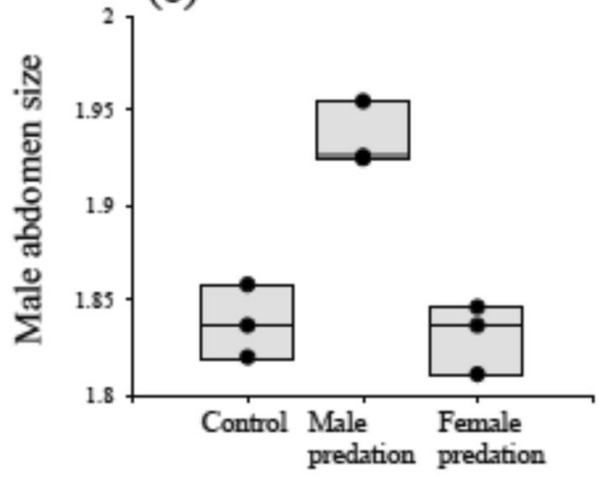

(c)

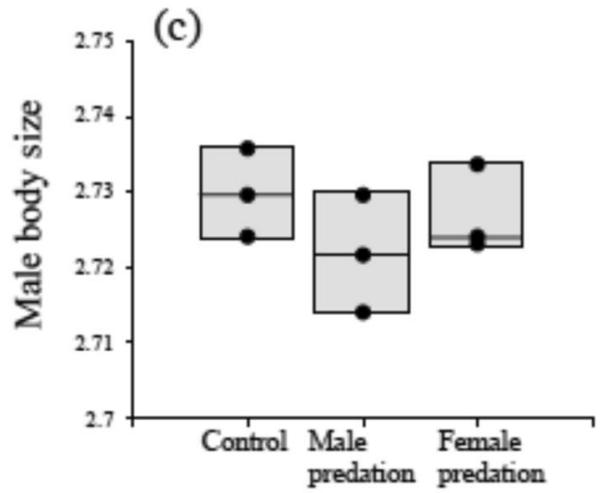

(d)

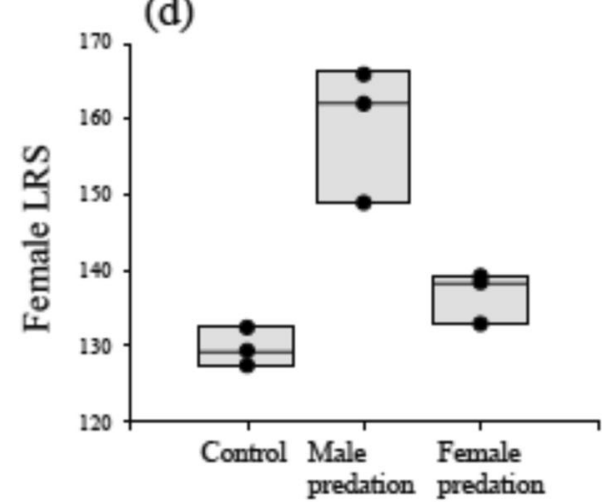

(e)
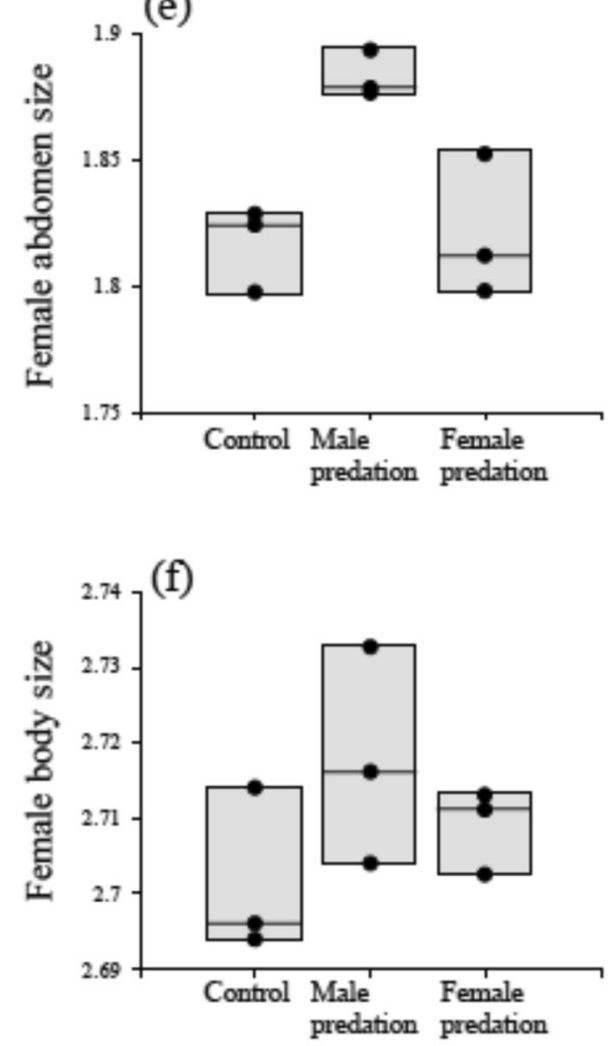

\section{Selection regime}

Fig. 2 Trait values after eight generations of experimental evolution. Replicate populations (3/treatment) were exposed to either male-only predation (middle columns), female-only predation (right-hand columns) or no predation (controls: left-hand columns), and effects on a range of traits was assessed. Traits were: Male mandible size $(\mathrm{mm})(\mathbf{a})$, Male abdomen size $(\mathrm{mg})(\mathbf{b})$, Male body size $(\mathrm{mg})$ (c), Female lifetime reproductive success (LRS: offspring number) (d), Female abdomen size (mg) (e), and Female body size ( $\mathrm{mg}$ ) (f) (shown are upper and lower quartile (the box) with medians (lines) and each dot represents the mean of one replicate population). Only populations evolve and thus population is the biologically relevant unit of replication in an experimental evolution study. Mean population values were estimated by measuring 20 individual males and female beetles/population. Source data are provided as a Source Data file.

can open up ecological space and lead to higher fitness of the prey's competitor taxa $\left.{ }^{64}\right)$. As we show, sex-biased predation can have analogous indirect effects intra-specifically (predation on males increases female fitness). However, the indirect impacts we document are unidirectional since female-biased predation does not alter female fitness or male sexual-trait size.

Micro-evolutionary responses to our treatments were not readily apparent until about four or five generations of selection, after which there was clear treatment-specific divergence. Non- linear responses like this are common in selection studies 65,66 (and see e.g., ${ }^{7,68}$ ) and there are many potential genetic and environmental mechanisms that can generate non-linearity. This includes genetic asymmetries (i.e., allele frequencies being unequal), differentially skewed genetic and environmental variation, non-additive genetic variation, and indirect selection ${ }^{65,66}$. We have no way of knowing the mechanistic cause of the patterns we document, but note that responses to directly selecting on mandible size previously revealed similar non-linearity ${ }^{39}$. There 


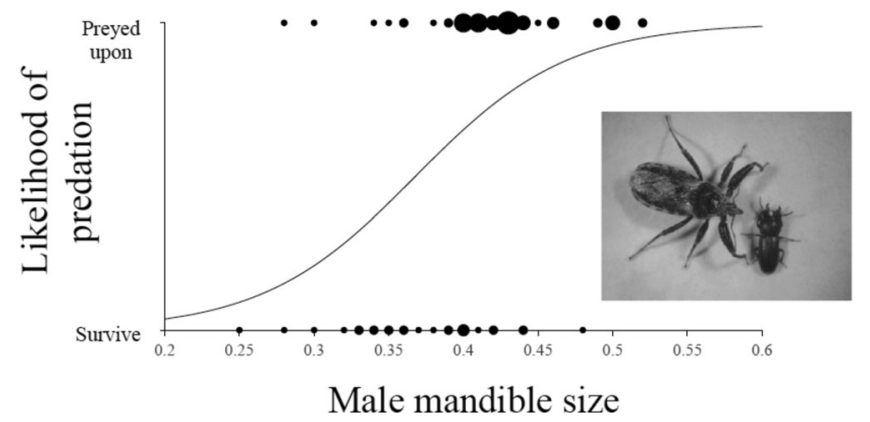

Fig. 3 The association between G. cornutus mandible size ( $\mathrm{mm}$ ) and likelihood of predation by A. venator. The graph shows that as mandible size increases so does the likelihood of predation. The curve is the fitted frequency from the GLM with dot size representing numbers (1-6) of individual $G$. cornutus (i.e., larger dots $=$ more beetles). Inset shows an $A$. venator about to attack a male $G$. cornutus. Note the size difference. Source data are provided as a Source Data file.

have also been many previous studies showing that males with smaller mandibles are less competitive and have lower fitness ${ }^{39,40}$, and that fighting ability is genetically correlated with mandible size $^{39}$. Consistent with these findings, males evolving with malepredation have smaller mandibles and are less adept fighters.

Overall this study provides direct evidence that predatormediated natural selection can evolutionarily reverse the exaggeration of a sexually selected trait. This finding is consistent with a vast body of fundamental theory ${ }^{5,6,69}$ and empirical evidence from observational and correlational studies ${ }^{19}$ (reviewed in the ref. ${ }^{1}$ ). We also reveal interesting outcomes when natural selection targets sex-limited sexually selected characters, since predator removal of a male-imposed conflict load increases female fitness. Thus sex-biased predation within a species can essentially mimic indirect ecological competition effects, as discussed above. Investigating the precise mechanistic detail and population level fitness effects of some of these findings is now required.

\begin{abstract}
Methods
Gnatocerus stock culture. The G. cornutus beetle culture originated from adults collected in Miyazaki City $\left(31^{\circ} 54^{\prime} \mathrm{N}, 131^{\circ} 25^{\prime} \mathrm{E}\right)$, Japan, and has been maintained in the laboratory of the National Food Research Institute, Japan, for about 50 years on whole meal enriched with yeast as food. The stock is made up of 1500-2000 beetles per generation and maintained in plastic cups (diameter $95 \mathrm{~mm}$, height $50 \mathrm{~mm}$ ) with a standing density of between 300 and 400 beetles per cup (for a more detailed description of the stock culture, see ref. ${ }^{70}$ ). This beetle is a stored product pest, and thus the laboratory conditions very closely mimic what have become their natural conditions. Virgin males and females were removed from the stock population as final instar larvae. Each larva was placed in one well of a 24-well tissue culture plate (Cellstar; Greiner Bio-One, Frickenhausen, Germany) until adult eclosion because pupation in G. cornutus is inhibited under high larval density ${ }^{70}$. After eclosion, both sexes were allowed to sexually mature for a period of 14 days prior to their use. We performed all rearing and experiments in a chamber maintained at $25^{\circ} \mathrm{C}$, $60 \%$ relative humidity, and with a photoperiod cycle of 14:10 h light/dark. All experiments in this study follow this protocol unless stated otherwise.
\end{abstract}

The predator. The assassin bug Amphibolus venator is predator of stored-product insect pests and preys on various stored-product insect pests including flour beetles ${ }^{48,71,72}$. These predators are frequently found in stored product facilities, which are the habitat of G. cornutus ${ }^{72}$ (and see Fig. 3). The A. venator culture originated from adults collected in a storehouse in Urasoe City, Okinawa, Japan, and has been maintained in the laboratory for about 5 years. The stock was initiated and maintained at 200 bugs per generation and housed in plastic containers $(230 \mathrm{~mm} \times 150 \mathrm{~mm} \times 80 \mathrm{~mm})$ with a standing density of between 30 and 40 bugs per cup. Each nymph was given an excess of food (seven final instar larvae of G. cornutus per week). Each adult female was allowed to mate with a male chosen randomly and to lay eggs in order to maintain the predator stocks. The predatory behavior of A. venator follows a stereotypical sequence: they recognize, chase, and capture prey (here G. cornutus) using their enlarged forelegs (similar to praying mantis $)^{48,73}$ $\underline{\text { Sexual selection }}$

Male predation
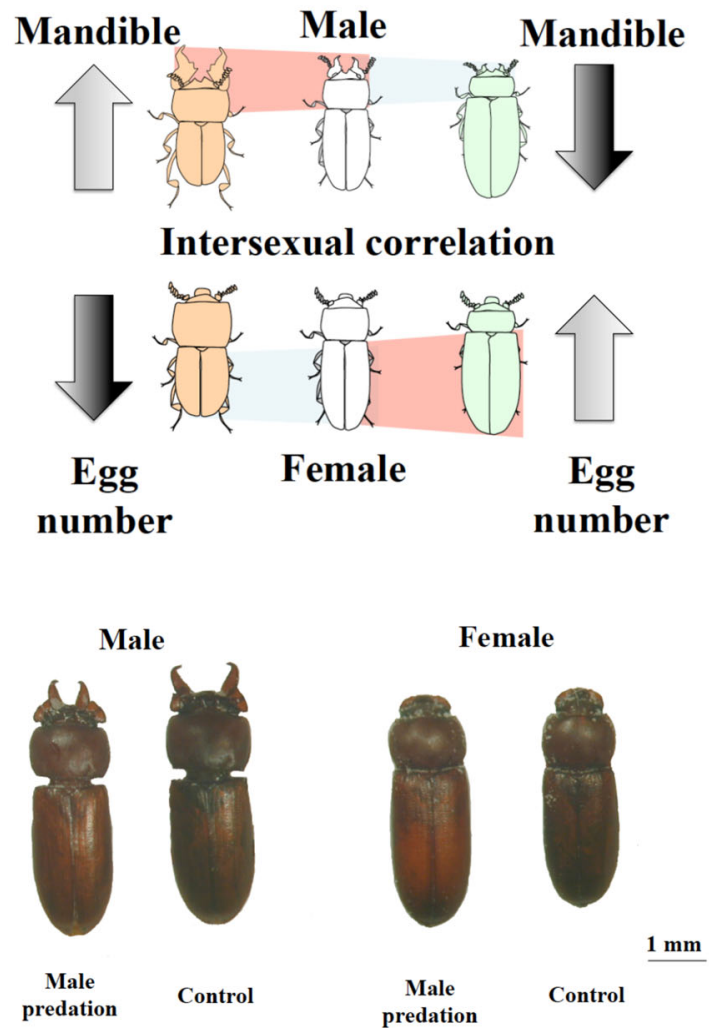

Fig. 4 The phenotypes after experimental evolution. The upper panel is a diagrammatic representation of the male and female phenotypes resulting from male-limited predation in comparison to those resulting from sexual selection on males. Sexual selection (left images) results in enlarged male mandibles, which require a masculinized head and prothorax to operate effectively. This fore-body masculinization results in a smaller male abdomen and because of intersexual correlations for abdomen size, a smaller female abdomen and capacity for fewer eggs, even though females never develop mandibles. Male-limited predation selects against the masculinized phenotype, ultimately resulting in larger male and female abdomens, and hence more eggs and higher fitness females (images on the right). The lower plate shows beetles from the male predation and control treatments and reveals the impact of evolving with male predation described above-both males and female evolve more feminized phenotypes (smaller mandibles for males and larger abdomen for both) compared to the controls (NB controls and female-predation have similar phenotypes (Figs. 1 and 2) hence we show only controls for clarity). Images were created by us.

\section{Gnatocerus breeding design and estimation of quantitative genetic para-} meters. Using a full sib/half sib experimental design, males (sires) $(N=35)$ were randomly assigned to three virgin females (dams) (all collected from the stock population). Pairs were housed in a plastic container $(17 \mathrm{~mm}$ diameter, $20 \mathrm{~mm}$ high) containing filter paper (17 mm diameter), and successful copulation was indicated by a stable end-to-end connection between the male and female. After mating, dams were immediately removed and individually placed in a plastic cup (70 $\mathrm{mm}$ diameter, $25 \mathrm{~mm}$ high) containing excess food $(20 \mathrm{~g})$. Each female was housed thus for two months to obtain offspring. All offspring from each female were reared to final instar (approximately 8 weeks). Three sons and three daughters per dam per sire (all pairings produced sufficient young) were haphazardly chosen for measurement of male traits (mandible, body, and abdomen size) and female traits (LRS, body, and abdomen size) at 14 days after eclosion $(N=315$ per sex) (trait measurement protocols below). Although we were primarily interested in the genetics of male mandible size and female fitness, we included both abdomen size and total body size measures. This is because previous work ${ }^{40}$ found that directly selecting for larger mandibles caused a correlated decrease in abdomen size, but had no effect on total body size. This suggests a trade-off between abdomen and prothorax size and sexual conflict over body shape. 


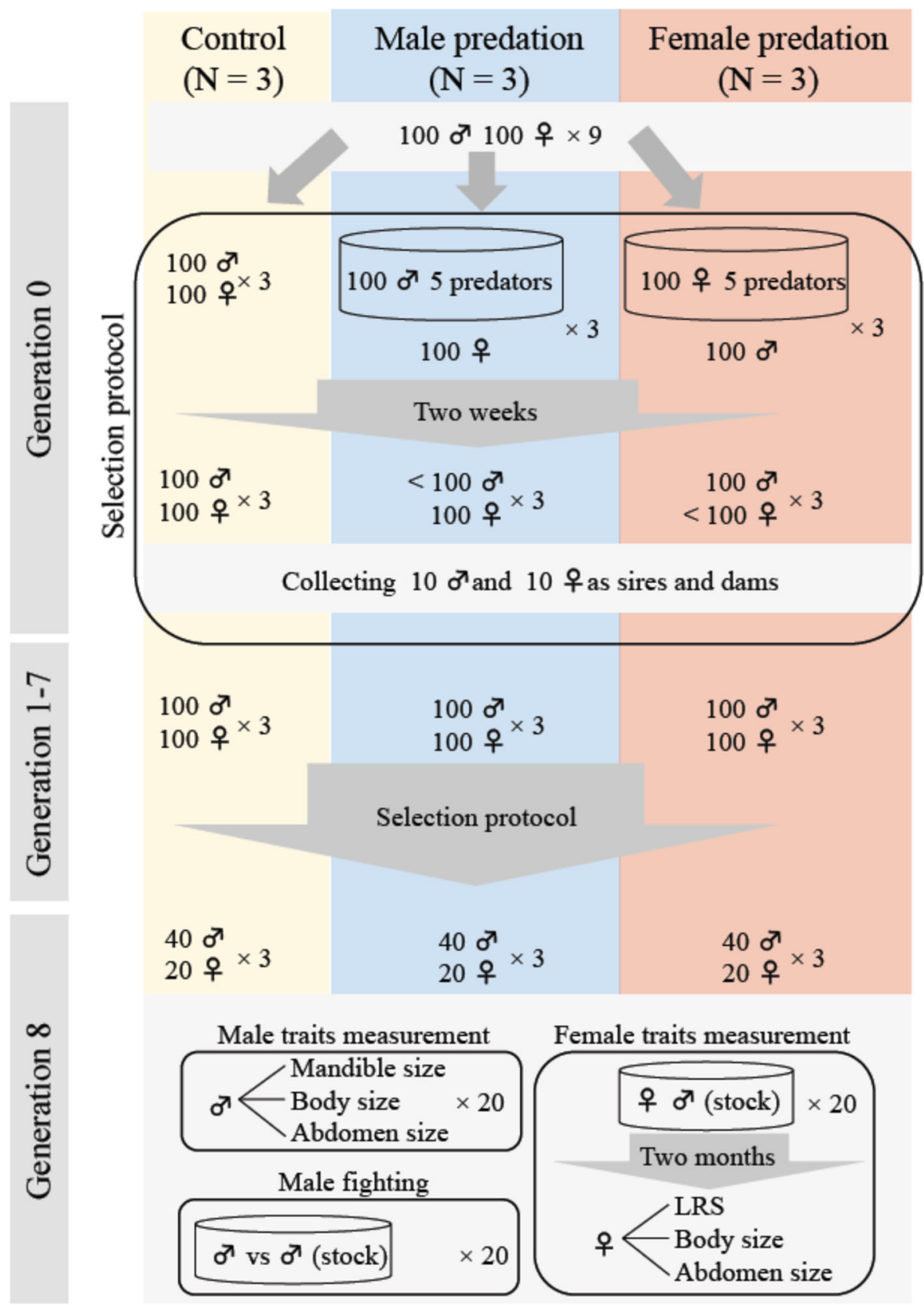

Fig. 5 A diagrammatic representation of the experimental evolution protocol. Included is the fighting assay after eight generations of experimental evolution. $N$ refers to the number of replicate populations/treatment.

Data from the breeding design were then analysed using pedigree-based animal models fit in ASReml- $\mathrm{R}^{74}$. First, to confirm the presence of additive genetic variance in each trait we fit a series of univariate animal models to (male) mandible, body and abdomen size, and to female (LRS). For each trait we compared the model fit to a reduced model with no additive genetic effects using a likelihood ratio test (LRT; adjusted for boundary conditions following ${ }^{75}$ ). We elected to combine male and female records for both body size and abdomen sizes as additional modeling provided little support for genotype-by-sex interactions (see "Results" section). However, for these traits a fixed effect of sex was included, as well as the (random) additive genetic effect since exploratory analysis showed sexual dimorphism in both traits (body size, males are $0.020 \mathrm{mg}$ (SE 0.005) larger on average, $t=3.58, P<0.001$; abdomen size, males are $0.019 \mathrm{mg}$ (SE 0.008) smaller on average, $t=2.463, P=0.014)$ ).

We then fitted a multivariate animal model to estimate genetic correlation $\left(r_{\mathrm{G}}\right)$ structure among the four traits with fixed effects of sex on body size, abdomen size, as well as their heritability $\left(h^{2}\right)$. The residual covariance structure was modeled as an unstructured matrix (but note residual covariance between the sex-limited traits of male mandible size and female LRS is not estimable from the data so was fixed to zero). We also ran reduced multivariate models with i) no genetic effects at all, and ii) a diagonal genetic variance matrix (i.e., genetic variance modeled on all traits but all genetic correlations assumed to equal zero) for comparison to the full model by LRT. This allows statistical inference at the level of the multivariate phenotype. We used estimated standard errors (SE) as a guide to nominal significance of pairwise genetic correlations (assuming approximate $95 \%$ CI are given by $r_{\mathrm{G}} \pm 1.96 \mathrm{SE}$ ).

Gnatocerus experimental evolution protocol-sex specific predation. We first collected 900 male and 900 female G. cornutus from the stock culture and haphazardly generated nine groups of 100 males and 100 females to establish three male-predation populations, three female-predation populations and three control (no predation) populations (generation 0) (Fig. 5). To simulate predation, 100 males (or females) were housed in a plastic container $(150 \mathrm{~mm}$ diameter, $50 \mathrm{~mm}$ high) containing an excess of beetle food $(45 \mathrm{~g})$. Then, five adult female A. venator (20-35 day olds) were randomly collected from the predator culture and placed into the container and the males (females) were exposed to them for two weeks. We then selected ten of the males (females) that survived the 2 weeks to act as sires (dams) of the predation treatments - ten opposite sex 
individuals were also taken/population to act as the non-selected dams (sires) that were not exposed to predation. We note that survival rate during this predation protocol was approximately $20 \%$. To propagate control populations, ten males and ten females were haphazardly selected per population to act as sires and dams. For each population/treatment the ten males and females were placed in a plastic cup (diameter $95 \mathrm{~mm}$, height $50 \mathrm{~mm}$ ) with $70 \mathrm{~g}$ of medium for 2 months, with males able to mate with females and females were allowed to lay eggs, until final instar larvae were obtained. Final instar larvae were collected to obtain the adults for subsequent generations. When the adults reached 14 days old, 100 males and 100 females per population were randomly selected to (potentially) seed the next generation, and in the predation treatments, exposed to predators as above. We then selected surviving animals as above and repeated for eight generations. Additionally, we also collected 50 males and 50 females per population from generation 1 to 7 to assess mandible, abdomen and body size responses to selection. At generation 8, 20 males and 20 females per population were haphazardly collected for measurement of male traits (mandible, body, and abdomen size) and female traits (LRS, body and abdomen size $)(N=180$ per sex $)$ (trait measurement protocols below).

Fighting ability. We also conducted male-male fights between evolved males and males from stock populations (Fig. 5). Briefly, after generation eight of experimental evolution, males from the nine populations (control, male predation, and female predation) were collected. We observed 20 contests per population $(n=180$ in total) in which focal experimental males competed against a male collected from the stock culture using standard methods ${ }^{39,76}$. In brief, the stock males were marked with white spots [Mitsubishi Paint-Marker] on their elytra for identification. Males 14 days old (after final eclosion) were used for the experiments. Before pairing, males were weighed with the electronic balance. To control for the effect of body size on fighting success, males were paired so that the difference in body size between contestants was less than $0.05 \mathrm{mg}$. Pairs were placed on filter-paper (17 mm diameter) in a plastic container (17 mm diameter, $20 \mathrm{~mm}$ high) and allowed to interact (and fight) for $1 \mathrm{~h}$. Previous work has shown that male fights occur in all trials when staged in this manner ${ }^{76}$. Males that pushed opponents and chased them were denoted the winner ${ }^{76}$. Trials were then continuously observed until fight outcomes could be scored.

Predation and mandible size. To more directly test for possible associations between predation and mandible size, seventy adult female A. venator (20-35 days old) were randomly collected from the stock culture and were individually aspirated into in a plastic dish $(90 \mathrm{~mm}$ diameter, $10 \mathrm{~mm}$ high) lined with a filter paper. After $30 \mathrm{~min}$, one male adult $\mathrm{G}$. cornutus collected from the stock culture (14 days old: mandible length measured prior to exposure) was added to each dish, and subsequent predation behavior was continuously observed for $30 \mathrm{~min}$.

Male trait measurement. We measured overall body mass and the posterior body mass (i.e., mesothorax, metathorax, and abdomen) as an abdomen size indicator ${ }^{40}$ (and see comments above. Briefly, each male was frozen at $-20{ }^{\circ} \mathrm{C}$ immediately after adult emergence. Mass measures were obtained to the nearest $0.01 \mathrm{mg}$ on an electronic balance (Mettler-Toledo AG, Laboratory and Weighing Technologies). The mandible length $( \pm 0.01 \mathrm{~mm})$ of each male was measured $( \pm 0.01 \mathrm{~mm})$ using a dissecting microscope monitoring system (VM-60; Olympus, Tokyo, Japan). Each specimen was positioned so that its longitudinal and dorsoventral axes were perpendicular to the visual axes of the microscope eyepiece (see ref. ${ }^{70}$ for landmarks).

Female trait measurement. To obtain LRS (lifetime reproductive success: our fitness proxy) each female ( 14 days of post-eclosure) was individually paired with a haphazardly selected male from the stock culture. After copulation, each female was maintained in a plastic cup ( $70 \mathrm{~mm}$ diameter, $25 \mathrm{~mm}$ high) containing excess food $(20 \mathrm{~g})$ for two months and allowed to lay eggs. This schedule was chosen because most eggs are laid by females within 2 months of mating ${ }^{77}$, and thus this is an accurate index of LRS ${ }^{42,78}$. To measure the LRS of each female, we counted all adults that emerged in the third month after pairing. After the laying period, each female was frozen at $-20^{\circ} \mathrm{C}$. Subsequently, the whole and posterior of the body (body size and abdomen size) were weighed with the electronic balance (as above).

Analysis. Apart from the genetic parameter estimation with an animal model (using ASReml-R as described above), all analyses were conducted using JMP for Windows version $8^{79}$. For the experimental evolution, we used population as the unit of replication ( $=9$ DF max.) with single fixed factor (with three levels: the experimental treatments) GLMs for each trait to test for effects of experimental evolution, with post-hoc testing for factor-level differences (note we did not have sufficient DF for a multivariate analysis). Results are as reported even after $\left(\right.$ conservative ${ }^{80}$ ) sequential Bonferroni correction. Fighting results were compared (as population rates of winning) using a generalized linear model (GLM) (JMP 7) ${ }^{76}$ To directly assess how mandible size affected predation probability we used a GLM with a binomial distribution, a logit-link function, and overdispersion test. Male mandible size was used as the explanatory variable, and predation impact (preyed upon $=1$, survive $=0$ ) was the response variable.

Reporting summary. Further information on research design is available in the Nature Research Reporting Summary linked to this article.

\section{Data availability}

The data that support the findings of this study are provided in Supplementary Data 1 This includes population mean trait values during and on completion of experimental evolution, fighting data, predation-mandible size data and the pedigree data. Source data are provided with this paper.

Received: 13 October 2020; Accepted: 17 May 2021; Published online: 08 June 2021

\section{References}

1. Andersson, M. 1994. Sexual Selection (Princeton University Press, 1994)

2. Shuster, S. M. \& Wade, M. J. Mating Systems and Strategies (Princeton University Press, 2003).

3. Andersson, M. \& Simmons, L. W. Sexual selection and mate choice. Trends Ecol. Evol. 21, 296-302 (2006).

4. Hosken, D. J. \& House, C. M. Sexual selection. Curr. Biol. 21, R62-R65 (2011)

5. Lande, R. Models of speciation by sexual selection on polygenic traits. Proc. Natl Acad. Sci. USA 78, 3721-3725 (1981).

6. Kirkpatrick, M. Sexual selection and the evolution of female choice. Evolution 36, 1-12 (1982)

7. Fisher, R. A. The Genetical Theory of Natural Selection (Claredon Press, 1930).

8. Prum, R. O. The Lande-Kirkpatrick mechanism is the null model of evolution by intersexual selection: implications for meaning, honesty, and design in intersexual signals. Evolution 64, 3085-3100 (2010).

9. Arnold, S. J. Mate Choice (ed. Bateson, P.) 67-108 (Cambridge University Press, 1983)

10. Pocklington, R. \& Dill, L. M. Predation on females or males: who pays the price for bright male traits? Anim. Behav. 49, 1122-1124 (1995).

11. Zuk, M. \& Kolluru, G. R. Exploitation of sexual signals by predators and parasitoids. Qrt. Rev. Biol. 73, 415-438 (1998).

12. Cade, W. H. Acoustically orienting parasitoids: fly phonotaxis to cricket song Science 1890, 1312-1313 (1975).

13. Ryan, M. J. Tuttle, M. D. \& Rand, A. S. Bat predation and sexual advertisement in a neotropical frog. Am. Nat. 119, 136-139 (1982).

14. Sakaluk, S. K. \& Belwood, J. J. Gecko phonotaxis to cricket calling song: a case of satellite predation. Anim. Behav. 32, 659-662 (1984).

15. Ryan, M. The Tungara Frog (University of Chicago Press, 1985)

16. Hosken, D. J., Bailey, W. J., O'Shea, J. E. \& Roberts, J. D. Localisation of insect calls by the bat Nyctophilus geoffroyi (Chiroptera: Vespertilionidae): a laboratory study. Aust. J. Zool. 42, 177-184 (1994).

17. Rotenbury, J. T., Zuk, M., Simmons, L. W. \& Hayes, C. Phonotactic parasitioids and cricket song structure: an evaluation of alternative hypotheses. Evol. Ecol. 10, 233-243 (1996).

18. Gray, D. A. \& Cade, W. H. Sex, death and genetic variation: natural and sexual selection on cricket song. Proc. R. Soc. Lond. B 266, 707-709 (1999)

19. Zuk, M., Rotenberry, J. T. \& Tinghitela, R. M. Silent night: adaptive disappearance of a sexual signal in a parasitized population of field crickets. Biol. Lett. 2, 521-524 (2006).

20. Pascoal, S. et al. Rapid convergent evolution in wild crickets. Curr. Biol. 24, 1369-1374 (2014)

21. Kotiaho, J. S. Costs of sexual traits: a mismatch between theoretical considerations and empirical evidence. Biol. Rev. 76, 365-376 (2001).

22. Lima, S. L. \& Dill, L. M. Behavioral decisions made under the risk of predation: a review and prospectus. Can. J. Zool. 68, 619-640 (1990).

23. Sakaluk, S. K. in Insect Defences. Adaptive Mechanisms and Strategies of Prey and Predators (eds. Evans D. L. \& Schmidt, J. O.) 63-90 (SUNY Press, 1990).

24. Candolin, U. Reproduction under predation risk and the trade-off between current and future reproduction in the threespine stickleback. Proc. R. Soc. B 265, 1171-1175 (1998).

25. Kotiaho, J. S., Alatalo, R. V., Mappes, J., Parri, S. \& Rivero, A. Male mating success and risk of predation in a wolf spider: a balance between sexual and natural selection? J. Anim. Ecol. 67, 287-291 (1998).

26. Zuk, M., Simmons, L. W. \& Cupp, L. Calling characteristics of parasitized and unparasitized populations of the field cricket Teleogryllus oceanicus. Behav. Ecol. Sociobiol. 33, 339-343 (1993).

27. Millar, N. P., Reznick, D. A., Kinnison, M. T. \& Hendry, A. P. Disentangling the selective factors that act on male colour in wild guppies. Oikos 113, 1-12 (2006). 
28. Reznick, D. A., Bryga, H. \& Endler, J. A. Experimentally induced life-history evolution in a natural population. Nature 346, 357-359 (1990).

29. Magnhagen, C. Predation risk as a cost of reproduction. Trends Ecol. Evol. 6, 183-186 (1991).

30. Cooper, W. E. Jr, Vitt, L. J., Hedges, R. \& Huey, R. B. Locomotor impairment and defense in gravid lizards (Eumeces laticeps): behavioral shift in activity may offset costs of reproduction in an active forager. Behav. Ecol. Sociobiol. 27, 153-157 (1990).

31. Berglund, A. \& Rosenqvist, G. Reproductive costs in the prawn Palaemon adspersus: effects on growth and predator vulnerability. Oikos 46, 349-354 (1986).

32. Lee, S. J., Witterm, M. S., Cuthill, I. C. \& Goldsmith, A. R. Reduction in escape performance as a cost of reproduction in gravid starlings, Sturnus vulgaris. Proc. R. Soc. Lond. B 263, 619-624 (1996).

33. Barry, M. J. The costs of crest induction for Daphnia carinata. Oecologia 97, 278-288 (1994).

34. Clobert, J. et al. Trade-offs in phenotypic traits: endurance at birth, growth, survival, predation and susceptibility to parasitism in a lizard, Lacerta vivipara. Funct. Ecol. 14, 675-684 (2000).

35. Koskela, E. \& YlÖnen, H. Suppressed feeding in the field vole (Microtus agrestis): an adaptation to cyclically fluctuating predation risk. Behav. Ecol. 6, 311-315 (1995).

36. Lind, J. \& Cresswell, W. Determining the fitness consequences of antipredation behavior. Behav. Ecol. 16, 945-956 (2005).

37. Rice, W. R. \& Chippindale, A. K. Intersexual ontogenetic conflict. J. Evol. Biol. 14, 685-693 (2001).

38. Hosken, D. J., Archer, C. R. \& Mank, J. Sexual conflict. Curr. Biol. 29, R451-R455 (2019).

39. Okada, K. \& Miyatake, T. Genetic correlations between weapons, body shape and fighting behaviour in the horned beetle Gnatocerus cornutus. Anim. Behav. 77, 1057-1065 (2009).

40. Harano, T., Okada, K., Nakayama, S., Miyatake, T. \& Hosken, D. J. Intralocus sexual conflict unresolved by sex-limited trait expression. Curr. Biol. 20, 2036-2039 (2010).

41. Yamane, T., Okada, K., Nakayama, S. \& Miyatake, T. Dispersal and ejaculatory strategies associated with exaggeration of weapon in an armed beetle. Proc. R. Soc. B 277, 1705-1710 (2010).

42. Katsuki, M., Harano, T., Miyatake, T., Okada, K. \& Hosken, D. J. Intralocus sexual conflict and offspring sex ratio. Ecol. Lett. 15, 193-197 (2012b).

43. Okada, K., Miyanoshita, A. \& Miyatake, T. Intra-sexual dimorphism in male mandibles and male aggressive behavior in the broad horned flour beetle Gnatocerus cornutus (Coleoptera: Tenebrionidae). J. Insect Behav. 19, 457-467 (2006).

44. Okada, Y., Suzaki, Y., Miyatake, T. \& Okada, K. Effect of weapon-supportive traits on fighting success in armed insects. Anim. Behav. 83, 1001-1006 (2012).

45. Honěk, A. Intraspecific variation in body size and fecundity in insects: a general relationship. Oikos 66, 483-492 (1993).

46. Pennell, T. M., de Haas, F. J., Morrow, E. H. \& van Doorn, G. S. Contrasting effects of intralocus sexual conflict on sexually antagonistic coevolution. Proc. Natl Acad. Sci. USA 113, E978-E986 (2016).

47. Wilson, A. J. et al. An ecologist's guide to the animal model. J. Anim. Ecol. 79, 13-26 (2010).

48. Imamura, T., Murata, M. \& Miyanoshita, A. Biological aspects and predatory abilities of hemipterans attacking stored-product insects. Jpn Agric. Res. Quart. 42, 1-6 (2008).

49. Fuchikawa, T. \& Okada, K. Inter-and intra-sexual genetic correlations of exaggerated traits and locomotor activity. J. Evol. Biol. 26, 1979-1987 (2013).

50. Miyatake, T. et al. Pleiotropic anti-predator strategies, fleeing and feigning death, correlated with dopamine levels in Tribolium castaneum. Anim. Behav. 75, 113-121 (2008)

51. Arnqvist, G. \& Rowe, L. Sexual Conflict (Princeton University Press, 2005).

52. Bonduriansky, R. \& Chenoweth, S. F. Intralocus sexual conflict. Trends Ecol. Evol. 24, 280-288 (2009).

53. Chippindale, A. K., Gibson, J. R. \& Rice, W. R. Negative genetic correlation for adult fitness between sexes reveals ontogenetic conflict in Drosophila. Proc. Natl Acad. Sci. USA 98, 1671-1675 (2001).

54. Rostant, W. G., Kay, C., Wedell, N. \& Hosken, D. J. Sexual conflict maintains variation at an insecticide resistance locus. BMC Biol. 13, 34 (2015).

55. Kiyose, K., Katsuki, M., Suzaki, Y. \& Okada, K. Competitive males but not attractive males reduce female fitness in Gnatocerus cornutus. Anim. Behav. 109, 265-272 (2015).

56. Kokko, H. \& Brooks, R. Sexy to die for? Sexual selection and the risk of extinction. Ann. Zool. Fenn. 40, 207-219 (2003).

57. Doherty, P. F. et al. Sexual selection affects local extinction and turnover in bird communities. Proc. Natl Acad. Sci. USA 100, 5858-5862 (2003).
58. Jarzebowska, M. \& Radwan, J. Sexual selection counteracts the extinction of small populations of the bulb mites. Evolution 64, 1283-1289 (2010).

59. Lumley, A. J. et al. Sexual selection protects against extinction. Nature 522, 470-473 (2015)

60. Rice, W. R. Sexually antagonistic genes: experimental evidence. Science 256, 1436-1439 (1992)

61. Holland, B. \& Rice, W. R. Experimental removal of sexual selection reverses intersexual antagonistic coevolution and removes reproductive load. Proc. Natl Acad. Sci. USA 96, 5083-5088 (1999).

62. Martin, O. Y. \& Hosken, D. J. Reproductive consequences of population divergence through sexual conflict. Curr. Biol. 14, 906-910 (2004).

63. Nelson, E. H., Matthews, C. E. \& Rosenheim, J. A. Predators reduce prey population growth by inducing changes in prey behavior. Ecology 85, 1853-1858 (2004)

64. Paine, R. T. Food web complexity and species diversity. Am. Nat. 100, 65-75 (1966).

65. Falconer, D. S. Introduction to Quantitative Genetics 2nd edn (Longman, 1981).

66. Falconer, D. S. Introduction to Quantitative Genetics 3rd edn (Longman, 1989).

67. Miller, G. T. \& Pitnick, S. Sperm-female coevolution in Drosophila. Science 298, 1230-1233 (2002).

68. Dobler, R. \& Hosken, D. J. Responses to selection and realized heritability of sperm length in the yellow dung fly (Scathophaga stercoraria). Heredity 104, 61-66 (2010)

69. Hall, D. W., Kirkpatrick, M. \& West, B. Runaway sexual selection when female preferences are directly selected. Evolution 54, 1862-1869 (2000).

70. Okada, K. \& Miyatake, T. Plasticity of size and allometry in multiple sexually selected traits in an armed beetle Gnatocerus cornutus. Evol. Ecol. 24, 1339-1351 (2010)

71. Pingale, S. V. Biological control of some stored grain pests by the use of a bug predator, Amphibolus venator Klug. Indian. J. Entomol. 16, 300-302 (1954).

72. Nishi, A., \& Takahashi, K. Effects of temperature on oviposition and development of Amphibolus venator (Klug) (Hemiptera: Reduviidae), a predator of stored product insects. App. Entomol. Zool. 37, 415-418 (2002).

73. Konishi, K., Matsumura, K., Sakuno, W. \& Miyatake, T. Death feigning as an adaptive anti-predator behaviour: further evidence for its evolution from artificial selection and natural populations. J. Evol. Biol. 33, 1120-1128 (2020).

74. Butler, D. G., Cullis, B. R., Gilmour, A. R., Gogel, B. G. \& Thompson, R. ASReml-R Reference Manual Version 4 (VSN International Ltd, 2017).

75. Stram, D. O. \& Lee, J. W. Variance components testing in the longitudinal mixed effects model. Biometrics 50, 1171-1177 (1994).

76. Okada, K., Okada, Y., Dall, S. R. X. \& Hosken, D. J. Loser-effect duration evolves independently of fighting ability. Proc. R. Soc. B 286, 20190582 (2019).

77. Tsuda, Y. \& Yoshida, T. Population biology of the broad horned flour beetle Gnathocerus cornutus (F) (Coleoptera Tenebrionidae) I. Life table and population parameters. Appl. Entomol. Zool. 19, 129-131 (1984)

78. Katsuki, M., Okada, Y. \& Okada, K. Impacts of diet quality on life-history and reproductive traits in male and female armed beetle, Gnatocerus cornutus. Ecol. Entomol. 37, 463-470 (2012).

79. SAS Institute. JMP Release 8 (SAS Institute Inc., 2008).

80. Nakagawa, S. A farewell to Bonferroni: the problems of low statistical power and publication bias. Behav. Ecol. 15, 4044-4045 (2004).

\section{Acknowledgements}

We especially thank Takahisa Miyatake for generously supplying the predators we used from his stocks. We also thank Stuart Bearhop, Sasha Dall and Dave Hodgson for discussion. D.J.H. was supported by the Leverhulme Trust (RF-2015-001). K.O. Y.O were supported by grants from the Japan Society for the Promotion of Science (KAKENHI 18K0641700 (K.O.), 18H04815,19H04913 (Y.O.), 21H02540 (Y.O. and K.O.)) and M.K was supported by a Grant-in-Aid for JSPS Fellows (19J40100).

\section{Author contributions}

K.O., M.K., T.S., Y.O., M.D.S., and D.J.H. designed the research; K.O., M.K., K.K., Y.O., and M.D.S. conducted experiments; K.O., M.D.S., and A.J.W. analyzed data; KO produced initial images for figures; and K.O., M.K., A.J.W., and D.J.H. wrote the paper with assistance from all authors.

\section{Competing interests}

The authors declare no competing interests. 


\section{Additional information}

Supplementary information The online version contains supplementary material available at https://doi.org/10.1038/s41467-021-23804-7.

Correspondence and requests for materials should be addressed to D.J.H.

Peer review information Nature Communications thanks the anonymous reviewers for their contribution to the peer review of this work.

Reprints and permission information is available at http://www.nature.com/reprints

Publisher's note Springer Nature remains neutral with regard to jurisdictional claims in published maps and institutional affiliations. (c) (1) Open Access This article is licensed under a Creative Commons Attribution 4.0 International License, which permits use, sharing, adaptation, distribution and reproduction in any medium or format, as long as you give appropriate credit to the original author(s) and the source, provide a link to the Creative Commons license, and indicate if changes were made. The images or other third party material in this article are included in the article's Creative Commons license, unless indicated otherwise in a credit line to the material. If material is not included in the article's Creative Commons license and your intended use is not permitted by statutory regulation or exceeds the permitted use, you will need to obtain permission directly from the copyright holder. To view a copy of this license, visit http://creativecommons.org/ licenses/by/4.0/.

(C) The Author(s) 2021 